\title{
PLATE MOTION AND EARTH ORIENTATION
}

\author{
A. Mallama and M. Kao \\ Science Applications Research \\ 4400 Forbes Boulevard \\ Lanham, Maryland 20706
}

\begin{abstract}
Earth orientation series are linked to the terrestrial reference frame in which the observing site locations are measured. The effect of tectonic motion is a simple rotation for any given plate, but the overall effect depends on the distribution of sites. The magnitude of this motion is large enough to be evident in the data. For example, the coefficient of rotation for the North American plate around the Earth's Y-axis is -0.8 millarcseconds per year in the AM0-2 plate motion model of Minster and Jordan. The VLBI analysis system at NASA/GSFC for computing earth orientation series has recently been enhanced by including the Minster and Jordan model for a priori tectonic effects. Tests indicate that the weighted-root-mean-square residual of observations to the solution is decreased by using this model.
\end{abstract}

\section{INTRODUCTION AND DATA DESCRIPTION}

NASA's Crustal Dynamics Project (CDP) developed and began using Mark III VLBI during the 1970's to study earth orienation and plate tectonic motion. Earth orientation is the instantaneous position of the crust of the earth relative to its spin axis (i.e., the two orthogonal components of polar motion), and the phase of the earth's rotation (i.e., Universal Time). Since the earth's crust is composed of tectonic plates that are in motion relative to one another, it is clear that plate motion is connected to the earth orientation series.

The VLBI data reported on in this paper were collected in three series of experiments, CDP, IRIS, and MERIT. The CDP experiments are managed by NASA/GSFC. They are a heterogeneous set taken on a network of about 20 sites that span the North American, Eurasian, and Pacific plates. The IRIS experiments are managed by NGS for the purpose of monitoring earth orientation. This system provides UT1 and both components of 363

M. J. Reid and J. M. Moran (eds.), The Impact of VLBI on Astrophysics and Geophysics, 363-364.

(c) 1988 by the IAU. 
polar motion at 5 day intervals. The MERIT data are from 14 experiments conducted during 1980 that serve to link the CDP and IRIS data.

\section{PLATE MOTION MODEL AND RESULTS}

The model of Minster and Jordan, 1978, has been chosen to account for plate motion in the VLBI data. It is derived from a geophysical data set consisting of hundreds of spreading rates, transform fault azimuths, and earthquake slip vectors. The results from our VLBI data when solved for site motions agree in an overall way with the Minster and Jordan model. The absolute motion model AMO-2, which corresponds to no net rotation, has been implemented in our VLBI analysis system.

The result that best illustrates an improvement in the data fit comes from the comparison between sets of experiments involving sites on the Pacific and North American plates, which have a large relative motion. The wrms fit in the solution is 93.2 psec without the plate motion model and 89.6 psec with it. The improvement with the model included indicates that it has successfully accounted for site motion. In the case where all the CDP, IRIS, and MERIT data are combined in a global solution the fit is 88.0 psec without the plate motion model and 87.0 psec with it. In an rss sense the model removes 13 psec of noise.

\section{DISCUSSION}

In our long-term effort to refine the VLBI technique we have included many calibrations such as precession and nutation, ionospheric delay, and ocean loading. The addition of the plate motion model to our system is in the category of a geophysical calibration. The results of our tests show that the application of the model improves the fit between observed and calculated data. We expect that a plate motion model will also improve the consistency between different sets of earth orientation data taken on entirely different networks, VLBI and otherwise.

\section{REFERENCE}

Minster, J.B. and Jordan, T.H., 'Present Day Plate Motions,' J. Geophysical Research, 83, 5331-53, 1978. 